\title{
A Study of Stress Relaxation in AZ31 Using High-Energy X-ray Diffraction
}

\author{
W. Tang ${ }^{\mathrm{a}}$, K. L. Halm ${ }^{\mathrm{a}}$, D. R. Trinkle ${ }^{\mathrm{b}}$, M. K. A. Koker ${ }^{\mathrm{c}}$, U. Lienert ${ }^{\mathrm{e}}$, P. \\ Kenesei $^{\mathrm{d}}$, A. J. Beaudoin ${ }^{\mathrm{a}, *}$ \\ ${ }^{a}$ University of Illinois at Urbana-Champaign, \\ Mechanical Science and Engineering, \\ Urbana IL USA \\ ${ }^{b}$ University of Illinois at Urbana-Champaign, \\ Mechanical Science and Engineering, \\ Urbana IL USA \\ ${ }^{c}$ Cornell High-Energy Synchrotron Source, \\ Ithaca NY USA \\ ${ }^{d}$ Advanced Photon Source, Argonne National Laboratory, \\ Argonne IL USA \\ ${ }^{e}$ Deutsches Elektronen Synchrotron Photon Science \\ Hamburg, Germany
}

\begin{abstract}
The micro-plasticity of the Mg alloy AZ31 is explored through High-Energy X-ray Diffraction (HEXD). Through cyclic loading of the sample, a softening response is found to follow the resolved shear stress for basal slip. Stress relaxation is studied by applying an incremental elongation increase while continuously collecting images from a detector array. The rate exponent associated with a particular reflection is developed by evaluating the average lattice strain in the loading direction from the composite detector image collected at a particular point in time, and then following the time evolution. A distinct rate exponent is identified for grain orientations that have a propensity for slip on second-order pyramidal planes, highlighting the capability to capture the simultaneous action of different deformation mechanisms through
\end{abstract}

\footnotetext{
${ }^{*}$ Corresponding author

Email addresses: tangwenlixyz2008@gmail.com (W. Tang), katherine.halm@gmail.com (K. L. Halm), dtrinkle@illinois.edu (D. R. Trinkle), koker@cornell.edu (M. K. A. Koker), ulrich.lienert@desy.de (U. Lienert), kenesei@aps.anl.gov (P. Kenesei), abeaudoi@illinois.edu (A. J. Beaudoin)
} 
HEXD.

Keywords:

Mg alloy, High-energy X-ray Diffraction, stress relaxation, plasticity, cyclic micro-plasticity

\section{Introduction}

Modern alloy systems are quite complex, and the mechanical response at the mesoscale can be very rich. One pathway to gain deeper understanding of mechanical response at the mesoscale is through use of high-energy $\mathrm{x}$ ray radiation from synchrotron sources. Procedures are well established for evaluation of the lattice strain tensor and grain orientation using hard x-rays $[1,2,3,4,5,6]$. Different kinds and arrangements of detectors provide for a wide variety of experimental approaches. Combined with in situ loading, it is possible to probe the mechanical response of a metal or alloy at the scale of individual grains.

In this paper, we examine the elasto-viscoplastic response of the magnesium alloy AZ31B using High-Energy X-ray Diffraction (HEXD). This material serves as one example of many that provide a path to improved energy efficiency through weight reduction in structural design. Of relevance to the present work, this alloy has been widely studied in the literature and this broad base of past effort provides a foundation for posing the HEXD experimental procedure and interpreting the results. The contribution of this work is the demonstration that constitutive response associated with individual modes of slip may be identified through HEXD.

In the following, a brief survey of the constitutive response in $\mathrm{Mg}$ and alloys relevant to the current study is set forth with emphasis given to kinetics - including mechanisms of dislocation-solute interaction that lead to collective dislocation motion. Two experimental procedures are detailed; an incremental cyclic loading procedure provides for characterization of mechanical response about the elasto-plastic transition and a stress relaxation study enables for the separation of rate-dependence associated with different slip modes. The discussion relates the results of the experiments to mechanisms proposed in the literature and suggestions are set forth for advancing models of crystal plasticity. 


\subsection{Background}

The preferred slip mode in $\mathrm{Mg}$ is on the basal planes, and basal slip is considered to be 'athermal' in character (see [7] and references therein), though alloying may introduce a more thermal character $[8,9]$. In the case of $\mathrm{Mg}$ single crystals, the critical resolved shear stress (CRSS) for basal slip increases from roughly 1 to $1.5 \mathrm{MPa}$ as temperature was decreased from $295 \mathrm{~K}$ to $4 \mathrm{~K}$, and the rate sensitivity exponent is given as $m=0.0212$ at $295 \mathrm{~K}$ [10] - indicative of a rate insensitivity for basal slip. In contrast, non-basal slip appears to have a more thermal character. In a study of single crystals oriented for pyramidal slip, Ahktar and Teghtsoonian [11] demonstrated a decrease in the thermally activated component of the flow stress with alloying. Obara et al. [12] showed a temperature dependence of $\langle c+a\rangle$ slip on $\{11.2\}$ planes in $\mathrm{Mg}$ single crystals. Data presented in references $[7,13]$ indicate a tendency toward rate insensitivity at room temperature, with transition to a (positive) rate-sensitive response at higher temperature. Activation of nonbasal slip systems is offered as a reason for the increase in rate-sensitivity with temperature as observed in jump tests on AZ31 in [14].

The kinetics of slip in the AZ31 alloy - as well as the suggestion of many possible deformation mechanisms - may be found in the literature. Prasad and Rao [15], through analysis of stress-strain data for cast-homogenized AZ31 in the temperature range of $300^{\circ} \mathrm{C}$ to $550^{\circ} \mathrm{C}$, found three distinct stress exponents and associated these exponents with rate-controlling mechanisms through the activation energy: dislocation climb (4.26); grain boundary self diffusion (6.14); pyramidal slip with cross-slip as the rate controlling process (3.35). Karimi et al. [14], using jump tests over a range of temperature and work hardening state, demonstrate response ranging from rate-insensitivity to a stress exponent of 3 (or a strain rate sensitivity of 0.33 ). Proposed mechanisms (listed roughly in order of increasing strain rate sensitivity) are twinning \& basal slip, prismatic cross-slip, pyramidal cross-slip and glidecontrolled dislocation creep. The kinetics of stress relaxation in AZ31 were studied by Lukáč and Trujanova [16]. They suggest the thermally-activated process is glide on non-compact planes and annihilation of dislocations. Dynamic recovery is found in tensile deformation of fine-grained AZ31B produced by equal channel angular extrusion [17] and activity of non-basal dislocations arising from incompatibility provides explanation for the enhanced ductility [18]. In a related alloy (relevant to the present work), Miller [19] finds a stress exponent of 4.6 for room temperature creep of cast AZ91 and draws association with dislocation climb as the rate-controlling mechanism. 
To draw agreement between simulation results using crystal plasticity and experimental observations, it appears necessary to pose a relatively high non-basal slip activity - even at room temperature. Prediction of the change in anisotropic response (r-value) with temperature was achieved by including $\langle\mathrm{c}+\mathrm{a}\rangle$ slip on $\{11.2\}$ planes in a viscoplastic self-consistent (VPSC) model[7]. Muránsky [20] also noted $\langle c+a\rangle$ slip on $\{11.2\}$ second-order pyramidal planes. These authors also observed a difference in the partitioning of elastic strain between diffracting grains having $\{11.0\}_{\|}$and $\{10.0\}_{\|}$parallel $^{1}$ to the loading axis, corresponding to the difference in Schmid factor for $\{11.2\}$ slip for these orientations. Grain boundary sliding provides an alternative to $\langle\mathrm{c}+\mathrm{a}\rangle$ slip in explaining the decrease of anisotropy with temperature increase: $\langle\mathrm{c}+\mathrm{a}\rangle$ dislocations are observed, but only in a minority of grains [21]; favorable comparison between model and experiment may be achieved through introduction of grain boundary sliding as a deformation mode [22]. Wang et al. used an elasto-viscoplastic self-consistent model to demonstrate an appreciable effect of stress relaxation on the measurement of lattice strain in a neutron diffraction study of AZ31 [23].

There is evidence for collective dislocation motion in the $\mathrm{Mg}-\mathrm{Al}-\mathrm{Zn}$ alloy system. Agnew and Duygulu raise the possibility of solute interactions with moving dislocations [7]. The PLC effect was indicated in testing of hot rolled AZ31 through correlation of acoustic emission events with serrations in the stress-strain curve [24]. Corby et al. [25] observed serrated yield in AZ91 - and not in binary alloys of $\mathrm{Mg}-\mathrm{Al}$ and $\mathrm{Mg}-\mathrm{Zn}$. These authors propose a mechanism upon the interplay of $\mathrm{Al}$ and $\mathrm{Zn}$ : $\mathrm{Al}$ in solid solution dictates the formation of pile-ups and Zn facilitates prismatic slip and development of forest dislocations. Central to the mechanism of [25] is the subsequent activation of new sources that cut the unstable forest. This non-diffusional mechanism was further advanced in a study of acoustic emission during deformation of AZ91 by [26].

The practical interest in the AZ31 alloy has prompted studies using high energy x-ray diffraction. Aydiner et al. performed an in situ study of the stress in a growing twin and parent grain [27]. In the mapping of stress about a crack in AZ31, Oddershede et al. observed stress relaxation above the yield point [28], with relaxation at the highest load attributed to crack growth (see

\footnotetext{
${ }^{1}$ We will adopt the notation of Muránsky etal. [20] and use (hk.l) $\|$ to indicate the normal of an (hk.l) plane to the loading axis
} 
Figure 2 of reference [28]).

\subsection{Objective}

Our objective is to study the microplastic deformation associated with crystallographic slip in AZ31. Two distinct loading procedures were performed in situ to provide insight into the strength and kinetics associated with basal and non-basal slip modes:

1. cyclic incremental loading, performed about the elasto-plastic transition, was applied to study the onset of basal slip in an aggregate of grains;

2. after loading into the elasto-plastic transition, a small cross-head displacement was prescribed and the ensuing increase of lattice strain in the direction of loading, followed by relaxation, was measured through the tracking of individual far-field diffraction peaks for several reflections.

\section{Materials \& Method}

Samples were machined from $12.7 \mathrm{~mm}$ thick annealed AZ31B plate. The gage section of the samples had a square $2 \times 2 \mathrm{~mm}^{2}$ cross-section and $12 \mathrm{~mm}$ gage length. The samples were machined from the plate such that the dominant c-axis texture component lay perpendicular to the loading axis. The experiments were conducted in transmission and with in situ loading at beamline 1-ID of the Advanced Photon Source. The energy was $80.725 \mathrm{keV}$ (Au Kedge) and was monitored during the experiment. The beam size was $200 \mu \mathrm{m}$ and $100 \mu \mathrm{m}$ in the horizontal and vertical directions, respectively. This beamsize was chosen to strike a balance between the (manageable) tracking of an aggregate of grains while maintaining distinct diffraction spots from individual grains. Digital image correlation was used to guide re-positioning of the sample during loading under displacement control, as well as for evaluating the strain in the vicinity of the illuminated region of the gage section.

Three GE detectors having $2048 \times 2048$ pixels were placed at a distance of $2.3 \mathrm{~m}$ and provided an azimuthal coverage of roughly $260^{\circ}$ (Figure 1a). A fourth detector was placed at $1 \mathrm{~m}$ from the sample, and could be moved in-and-out of the beam. This single detector was used during the experiment for 'on-the-fly' indexing and maintaining sample position through tracking of diffraction spots. Initial calibration of the detector distance was carried out using $\mathrm{CeO}_{2}$ patterns and the FIT2D program [29]. 


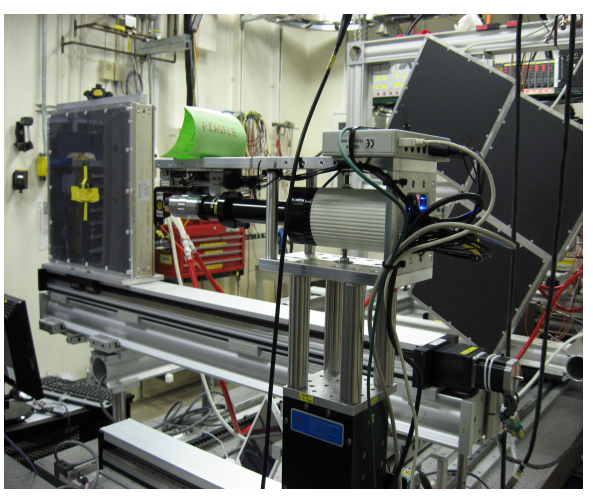

(a) detector arrangement

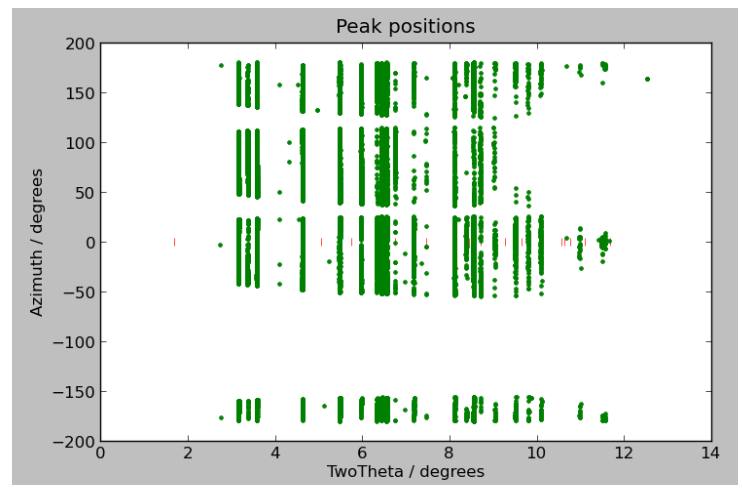

(b) identification of diffraction spots (ImageD11)

Figure 1: The 'hydra' detector array at Sector 1 of the Advanced Photon Source.
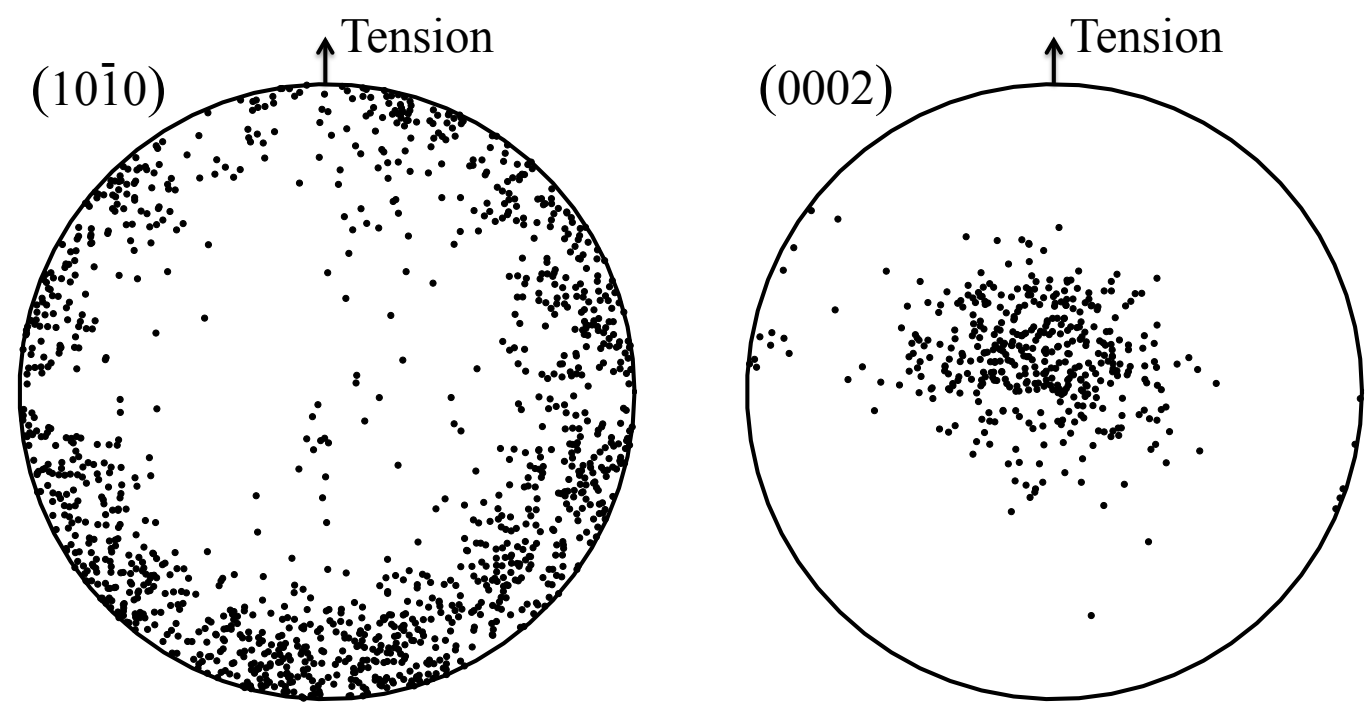

Figure 2: Pole figures for AZ31 plate with normal direction out of the page. 
The Fable software suite [30] was used for identifying diffraction peaks, generating diffraction vectors and refining the detector calibration. For analysis using the three-detector array, filtered peaks were first identified for individual detectors using the peaksearch program and these peak locations were then translated and rotated so as to be placed on a single virtual detector using a custom python script. The following steps are then the same for the virtual detector image or data collected from the single detector shown to the left of Figure 1a: the peak positions are read into the ImageD11 program of the Fable suite (Figure 1b); the transformation module used to generate "g" (diffraction) vectors; the GrainSpotter program is used for indexing of grains [31]. The peak positions developed from the hydra array are shown in Figure 1b. The coverage of the individual detectors is indicated by the Azimuthal (vertical) axis. Indication of the distance calibration may be seen through vertical alignment of the peaks associated with different reflections.

Pole figures providing the texture of the AZ31B are given in Figure 2. These pole figures were collected using a single detector during the stress relaxation study, to be detailed below, and are plotted in a conventional manner with the plate normal direction out of the page. The texture is typical of a rolled and annealed product [32]. The tension direction for the in situ loading is indicated at the top of the pole figures. The tensile loading axis is perpendicular to the basal texture (normal direction), and as such the contribution of the $\{10 \overline{1} 2\}$ twinning as a deformation mechanism should be negligible [7].

\subsection{Incremental Loading Study}

Incremental loading was performed by first loading the sample to a nominal stress level of $62 \mathrm{MPa}$, then the load was cycled using the profile shown in Figure 3.

\subsubsection{Analysis of Lattice Strain and Stress}

In the analysis of lattice strain, the dot product between the modelled and measured unit g-vectors had to be within 0.9995, and only Friedel pairs were included. Analysis of lattice strain follows the procedure given in [33] and is given in the appendix for completeness. Typically, more than 80 g-vectors were identified for the measurements from cycling. For the last three load steps, the number of $\mathbf{g}$-vectors identified for the strain calculation decreased. Stress was developed from lattice strain using moduli for pure $\mathrm{Mg}$, taken from reference [34] and listed in Table 1. 


\begin{tabular}{|c|c|c|c|c|}
\hline$C_{11}$ & $C_{33}$ & $C_{44}$ & $C_{12}$ & $C_{13}$ \\
\hline 59.3 & 61.5 & 16.4 & 25.7 & 21.4 \\
\hline
\end{tabular}

Table 1: Moduli used for calculation of stress (GPa), from [34]. 


\subsection{Relaxation Test}

The stress relaxation was carried out in an 'open loop' fashion. The sample was slowly loaded to point $A$, as shown in Figure 4. Then, sampling of detectors was initiated at 5 frames per second, followed by a cross-head displacement increment. During the course of the loading and relaxation, the position of the sample relative to the beam was maintained using DIC (digital image correlation) and cross-check by (vertical) scanning of a diffracting peak from a particular grain. Repositioning within $2 \mu \mathrm{m}$ was routinely achieved through use of DIC.

Emphasis in the presentation of results will be on the relaxation step D indicated in Figure 4. This point is associated with significant relaxation, prior to spreading of diffraction spots due to significant plastic deformation (as observed for $\mathrm{E}$ ).

\subsubsection{Processing of Detector Images for Study of Relaxation}

As noted above, diffraction images were collected at a rate of 5 frames per second. Images from the three detectors were averaged in time using

$$
\bar{P}_{n}(x, y)=\frac{1}{3} \sum_{n-1}^{n+1} P_{i}(x, y)
$$

where $P_{i}$ is the raw image and $\bar{P}_{n}$ is the averaged image. The individual detector images are then rotated and mapped onto a virtual $2995 \times 2995$ 
detector. In this mapping, it is possible to have empty pixels in the virtual, target detector, as a consequence of the integer math. To develop a continuous image, a $5 \times 5$ Gaussian kernel was applied to source pixels of the three source detectors in the mapping,

$$
G(x, y)=\frac{1}{2 \pi \sigma^{2}} e^{-\frac{x^{2}+y^{2}}{2 \sigma^{2}}}
$$

where $\sigma$ is selected to minimize spreading, while filling the virtual detector image. The value used in the present work was 0.8 (pixels).

Peaks were isolated from the virtual detector image by first selecting a threshold level that drew a compromise between preserving higher order peaks and maintaining a separation of stronger peaks, then determining connected pixels through use of an ancillary binary image [35, 36]. Time history data for two peaks are provided in Figure 5 . These well-defined peaks exhibit a minimum of azimuthal spreading, indicative of a well-annealed microstructure. A peak along the vertical axis of the detector array, Figure 5a, shows significant radial motion during the loading increment, then relaxation of the stress (through an 'outward' motion of the peak), Figure 5c. The stepped response shown in the time series plot of radial motion was observed for some - but not all - peaks, and will be addressed below. In contrast to the marked motion of peaks along the loading direction, peaks with diffraction vectors perpendicular to the loading, Figure 5b, show much less motion.

\section{Results}

\subsection{Incremental Loading}

An aggregate of thirty-three grains were indexed and tracked through the incremental loading. The (0002) pole figure is shown in Figure 6a. The sample is loaded in the rolling direction (out of the page in Figure 6a).

The normal components of stress for two particular grains are shown in Figures $6 \mathrm{c}$ and $6 \mathrm{~d}$. Shear stress components were generally quite small. A softening behavior is evident for both of the grains. Examining the component of stress in the tension direction, $\sigma_{33}$, and drawing attention to the final series of loading steps from the minimum stress value to the maximum, it is clear that values for this final portion of the curve lie below the initial incremental steps (for corresponding strain). This indicates microplasticity through the course of an extended elasto-plastic transition. 


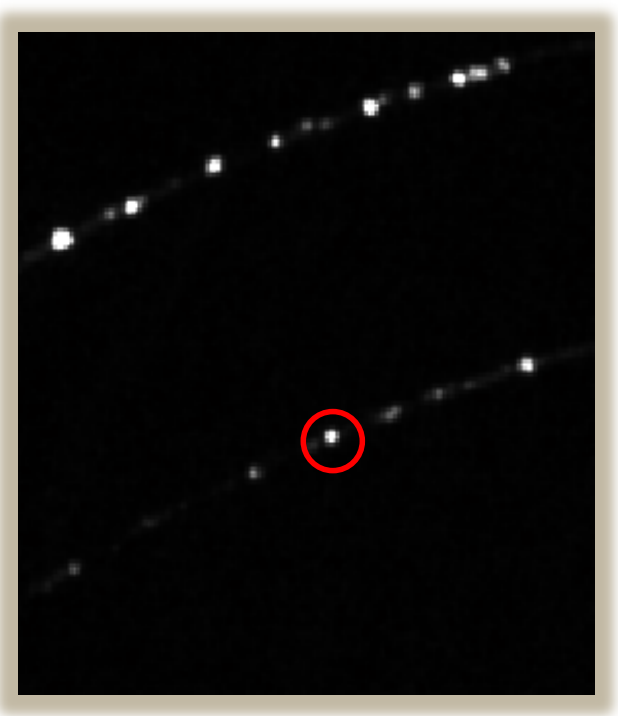

(a) $\eta=0$

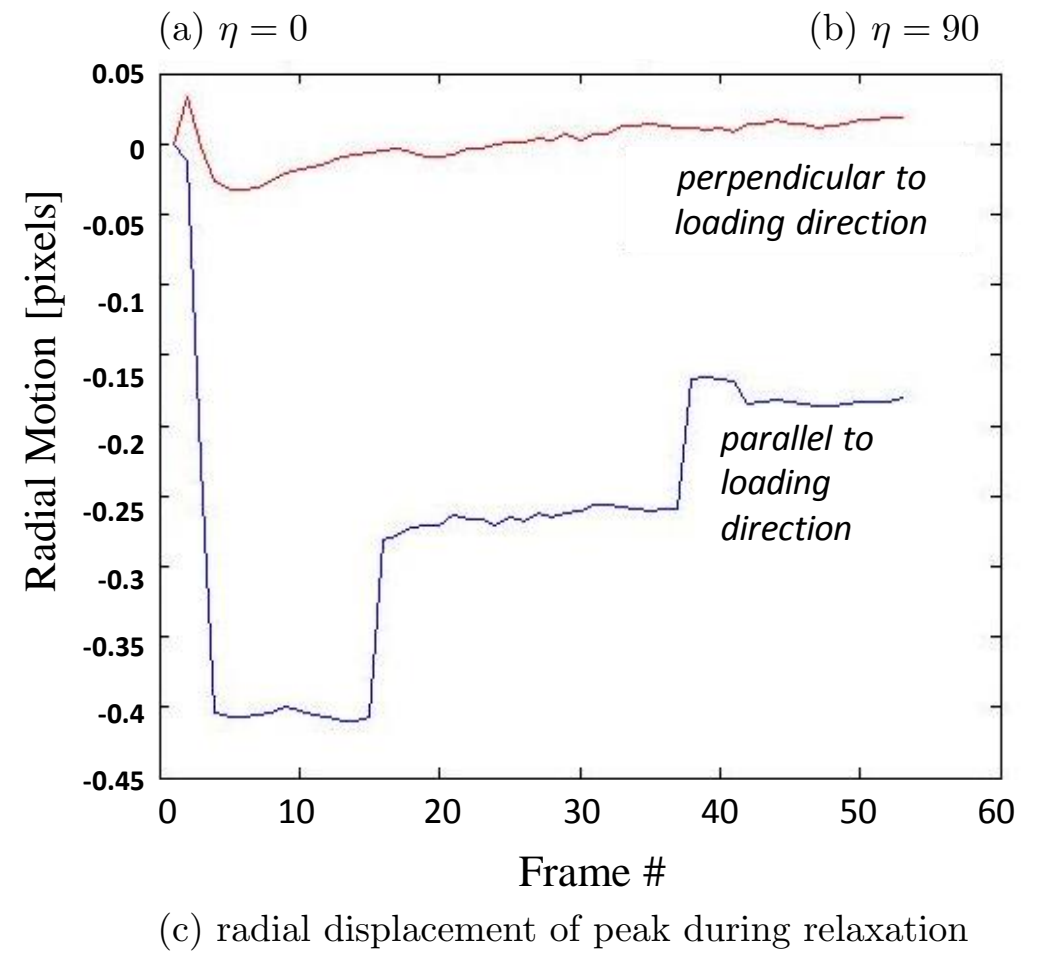

(c) radial displacement of peak during relaxation

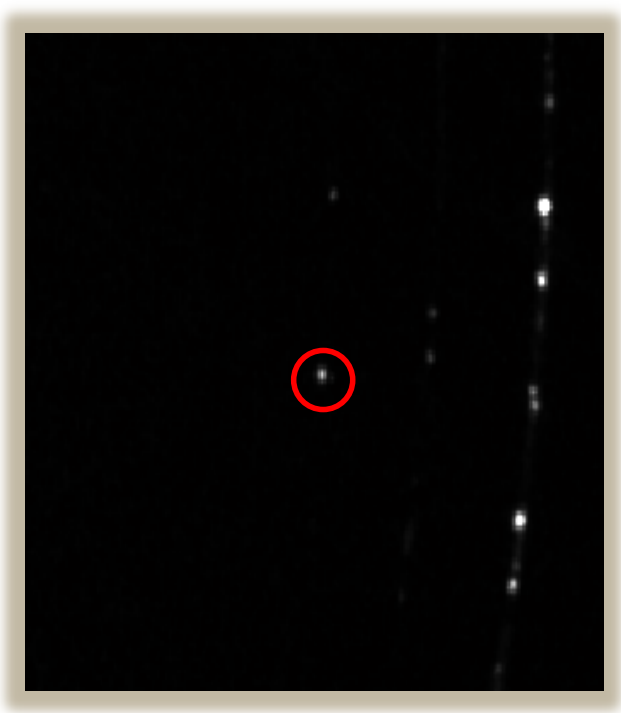

(b) $\eta=90$

Figure 5: Example diffraction spots from relaxation study: (a) along and (b) perpendicular to the loading direction. The circled diffraction spots are both from the (10.0) ring and tracked during relaxation. 
(a) (0002) pole figure, with tensile axis out of the page.

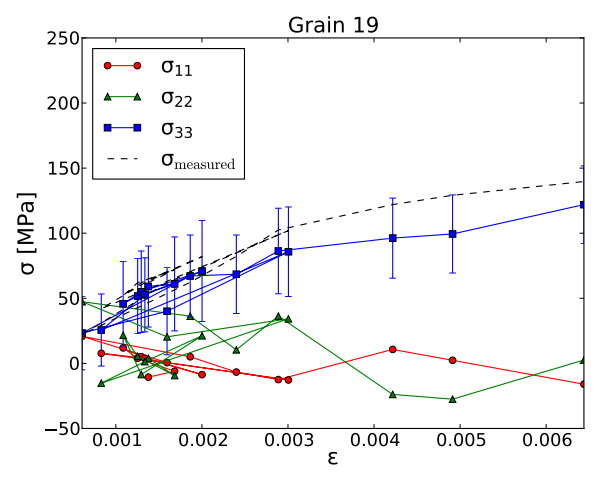

(c) lattice strain history for grain 19 (circled in the (00.2) pole figure)

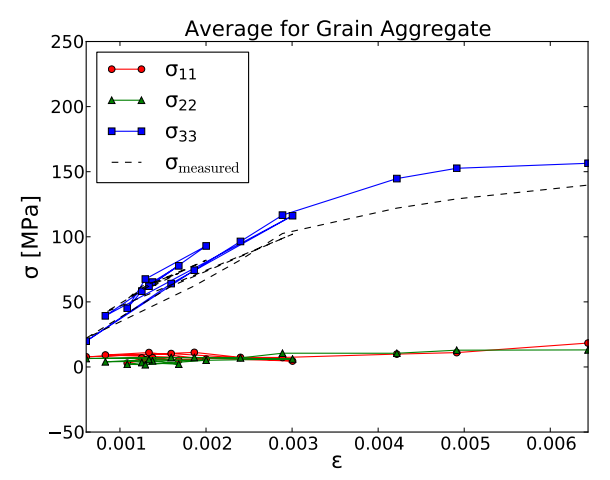

(b) aggregate response

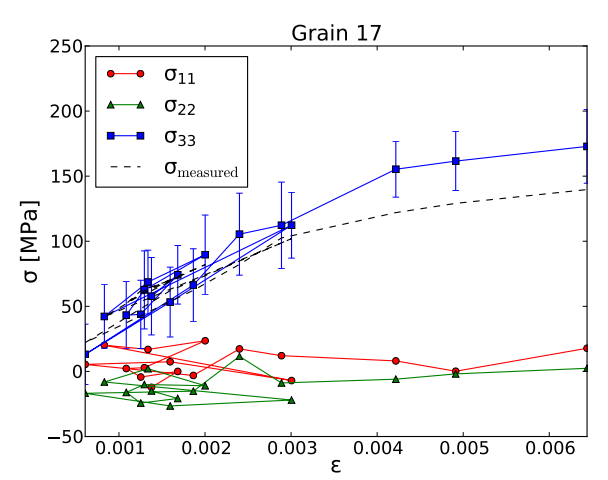

(d) lattice strain history for grain 17

Figure 6: Incremental Loading Study 


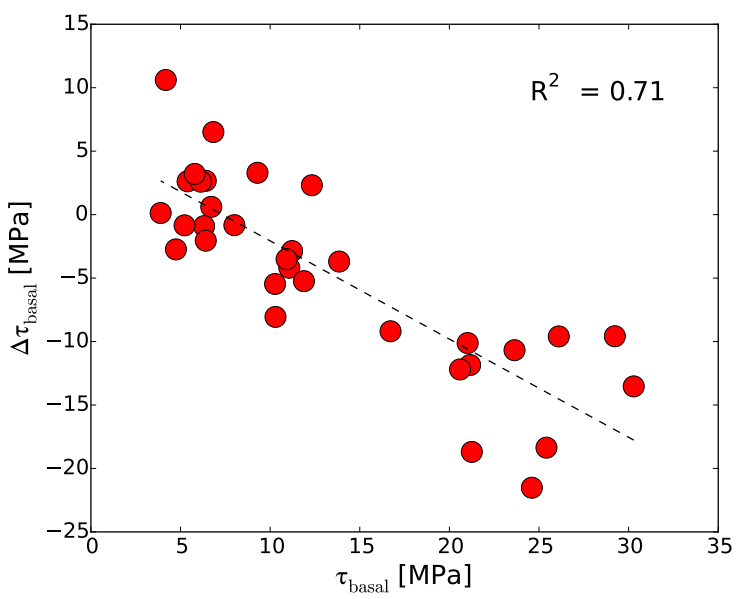

Figure 7: Softening response developed through cyclic testing.

The averaged response of all grains is given in Figure 6b. The nominal stress measured using the load cell is shown as a dashed line. Here again, a softening response is evident for the grain aggregate. Presuming that basal slip is the first mode to be activated, the maximum resolved shear stress, $\tau_{\text {basal }}$ is identified by transforming the stress to the lattice frame, computing the resolved shear stress for each of the basal slip systems and selecting the maximum value. The difference of the maximum resolved shear stress between the end of the third loading cycle and the initial loading (circled points in Figure 3),

$$
\Delta \tau_{\text {basal }}=\tau_{\text {basal }}^{(11)}-\tau_{\text {basal }}^{(0)}
$$

is plotted against $\tau_{\text {basal }}^{0}$ in Figure 7 (here the superscript denotes the loading step, as indicated in Figure 3). The decrease in stress appears to follow well the resolved shear stress on the basal plane.

\subsection{Stress Relaxation}

\subsubsection{Aggregate Response}

Peaks measured through use of the three detector array, projected on the single virtual detector as described above, are grouped by reflection to assess stress relaxation. The collection of peaks associated with a particular reflection is used to fit an ellipse through least squares minimization. The ellipse provides an averaged response of lattice strain along the loading direction, $\varepsilon_{z}$ with time. The time history for several reflections is shown in Figure 8a. 
Further insight may be gained through partitioning these curves into the individual phases of loading and relaxation. The peak value of the averaged strain for each reflection of Figure 8a, as developed during the initial loading phase and prior to relaxation, is plotted against the Schmid factor for basal slip in Figure 8b. A trend is evident, indicating a connection between the basal activity and loading response: orientations better suited for basal slip show reduced (elastic) lattice strain. Some plastic accommodation occurs during loading due to basal slip activity.

The net relaxation is found by simply subtracting the peak strain value from the time history,

$$
\Delta \varepsilon_{z}=\varepsilon_{z}(t)-\max \varepsilon_{z}(t) .
$$

The result is shown in Figure 8c. Standing out in this figure is the marked difference in response between the (10.0) and (11.0) reflections. These orientations are essentially identical when considering the resolved stresses for basal or prismatic slip. Difference lies, however, in the Schmid factor for pyramidal $\langle c+a\rangle$ slip on $\{11.2\}$ second order pyramidal planes. The Schmid factors for the $(10.0)_{\|}$and $(11.0)_{\|}$grains are 0.34 and 0.45 , respectively (see also Figure 4(e) of [20]). The largest relaxation response, seen in $(11.0)_{\|}$ orientations, appears to be a consequence of reduced basal activity upon loading, and - in turn - more elastic accommodation of the applied deformation, followed by relaxation as a consequence of favorable conditions for $\langle\mathrm{c}+\mathrm{a}\rangle$ slip.

$\mathrm{Li}$ [37], assuming constant 1) mobile dislocation density, 2) internal stress, $\sigma_{i}$, and 3) stiffness of the test system proposed the following relation between stress and time, $t$,

$$
\sigma-\sigma_{i}=K^{\prime}(t+a)^{\frac{-1}{m^{\star}-1}} .
$$

where $K^{\prime}, a, \sigma_{i}$ and $m^{\star}$ are fitting parameters. The parameter $m^{\star}$ follows from the empirical power law relationship for dislocation velocity, $v$,

$$
v=B \tau^{\star m^{\star}}
$$

where $\tau^{\star}$ is the effective shear stress and $B$ is the mobility. This relation is used in reference [16] to develop the internal stress $\sigma_{i}$ from stress relaxation data for AZ31. For the purposes of this study, the equation was modified through dividing by some 'effective' modulus for a reflection, $E_{\text {ref }}$, giving

$$
\varepsilon_{z}-\varepsilon_{i}=K^{\prime \prime}(t+a)^{\frac{-1}{m^{\star}-1}} .
$$




\begin{tabular}{|c||c|c|c|}
\hline Reflection & $\varepsilon_{i}\left(\times 10^{-4}\right)$ & $m^{\star}$ & error $\left(\times 10^{-4}\right)$ \\
\hline$(10.0)$ & 2.69 & 4.59 & 0.165 \\
$(11.0)$ & 1.97 & 2.96 & 0.257 \\
$(10.1)$ & 1.80 & 4.46 & 0.225 \\
$(20.1)$ & 2.09 & 4.43 & 0.153 \\
$(11.2)$ & 1.38 & 4.21 & 0.173 \\
\hline
\end{tabular}

Table 2: Parameters for fit of relaxation data for individual reflections.

where $K^{\prime \prime}=K^{\prime} / E_{\text {ref. }}$. The results of the fit are shown as dashed lines in Figure 8a and listed in Table 2. The error is computed as

$$
\mathrm{E}=\sqrt{\frac{\sum_{i=1}^{N}\left(\varepsilon_{\mathrm{z}}^{i}-\tilde{\varepsilon}_{\mathrm{z}}^{i}\right)^{2}}{N-4}}
$$

where $\varepsilon_{\mathrm{z}}^{i}$ and $\tilde{\varepsilon}_{\mathrm{z}}^{i}$ are the measured and predicted lattice strains, respectively. It is clear that eq. (7) serves well to describe the relaxation data following from the fitting of an ellipse. Examination of the effective rate sensitivity, $m^{\star}$, the result for $(11.0)_{\|}$stands out: the stress exponent $m^{\star}$ is roughly $3-$ significantly less than the value for other diffraction rings.

\subsubsection{Time History of Individual Diffraction Peaks}

In the observation of peaks during relaxation, sharp jumps in peak displacement (on the detector) were often observed. One example is shown in Figure 9. This is a (10.1) peak located on the upper detector, along the loading axis, taken during stress relaxation from relaxation step D (the focus of this paper). The motion of the peak center-of-mass (COM), Figure 9a, highlights distinct jumps during relaxation - as indicated in Figure 9a. This behavior was noted for many individual peaks examined in the study and during all of the stress relaxation steps; another example is the the (10.0) peak of Figure $5 \mathrm{c}$ observed during relaxation step A. For a more complete exposition, see the thesis by Tang [35].

Examination of the peak at different frames during the relaxation shows distinct changes in the peak; see Figure 10. This (10.1) peak is located along the vertical on the upper detector. The (subpixel) inward motion of the peak COM during loading is evident through visual comparison of images 1 and 2. There is also a clear difference between the distribution of intensity between images 1 and 3, along with (roughly) a pixel shift in the peak position. 

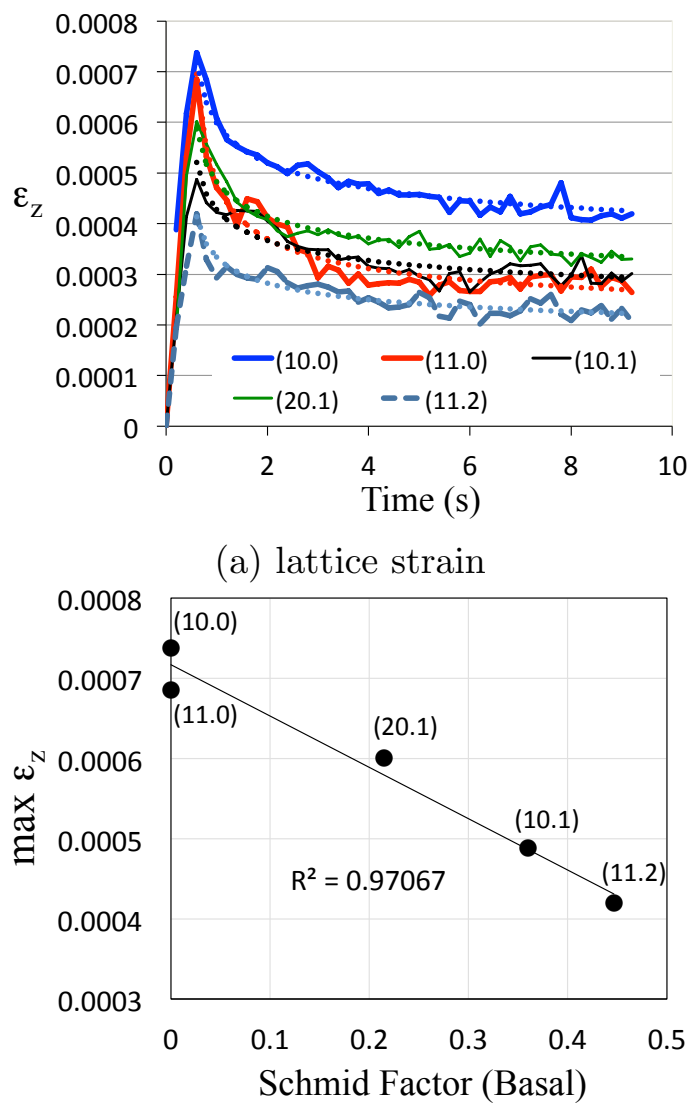

(b) peak lattice strain

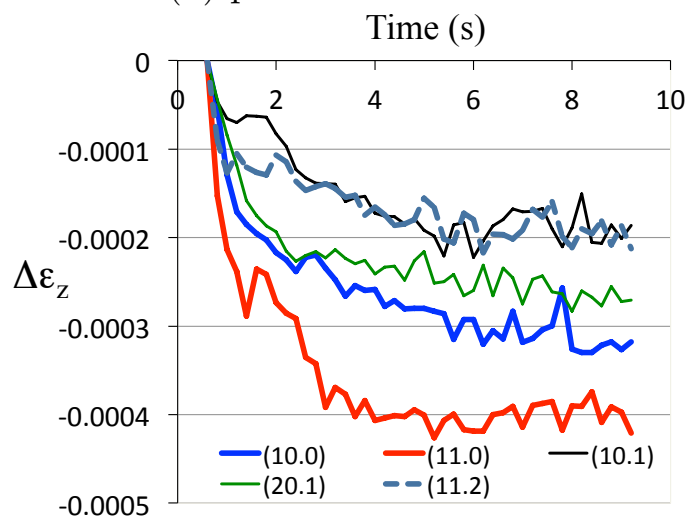

(c) relaxation

Figure 8: Relaxation of Lattice Strain 
The integrated intensity during relaxation is shown in Figure 9c. There is an abrupt increase in intensity associated with the jump in radial displacement. Again, similar behavior of correlated radial displacement and change in integrated intensity was observed for other peaks - with both increases and decreases in the intensity found. This suggests that some portion of the grain rotated in/out of the Bragg diffraction condition for a decrease/increase in intensity.

\section{Discussion and Conclusions}

The present work combines the results of two separate experiments using AZ31: incremental loading below the usual yield stress and stress relaxation. The complete lattice strain tensor was recovered for an aggregate of grains through the incremental loading. These results demonstrate that grains favorably oriented for basal slip undergo softening through the course of incremental loading - below macroscopic yield [18, 38]. This result is consistent with the the stress increase associated with displacement increments taken in the study of stress relaxation, as indicated in Figure 8b: the stress increase for a particular grain orientation is inversely proportional to the resolved shear stress on the basal slip plane. As stated by Wang et al. [23], "subsets of grains in soft orientations yield first and stop bearing internal stresses, while subsets of grains in hard orientations continue to deform elastically."

Several observations made in the neutron diffraction study of AZ31B by Muránsky et al. are reinforced by the present work. Their suggestion of load shedding for $\{10.2\}_{\|},\{10.1\}_{\|}$and $\{11.2\}_{\|}$orientations are supported by the time-resolved lattice strain increment shown in 8b. This (microplastic) deformation due to basal slip below the (macroscopic) yield point is observed on a grain-by-grain basis in the incremental loading study (7). These authors also point out that, close to yield, the $\{10.0\}_{\|}$orientation is 'plastically harder' than the $\{11.0\}_{\|}$orientation, on the basis of the Schmid factor favoring $\{11.2\}$ slip for the latter. In the present work, evidence for presence of $\{11.2\}$ slip is seen in the enhanced relaxation of $\{11.0\}_{\|}$oriented grains. The stress exponent, $m^{\star}$, recovered from the analysis of stress relaxation suggests a different rate-determining mechanism for $\{11.2\}$ slip, as compared to other modes of slip. This result holds implications for models of crystal plasticity: both the strength and rate-dependence should be considered in specification of the the slip system response, and the rate exponent may vary for different slip systems. 


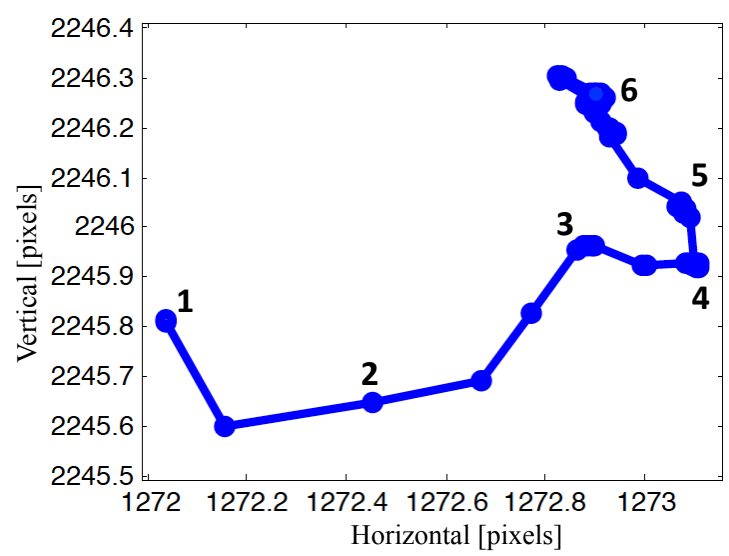

(a) center of mass location on detector (in pixel units)

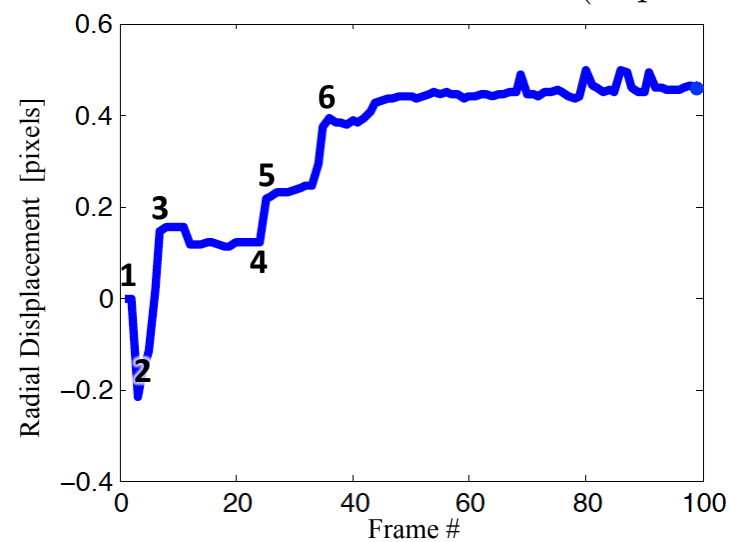

(b) radial displacement of center of mass (in pixel units)

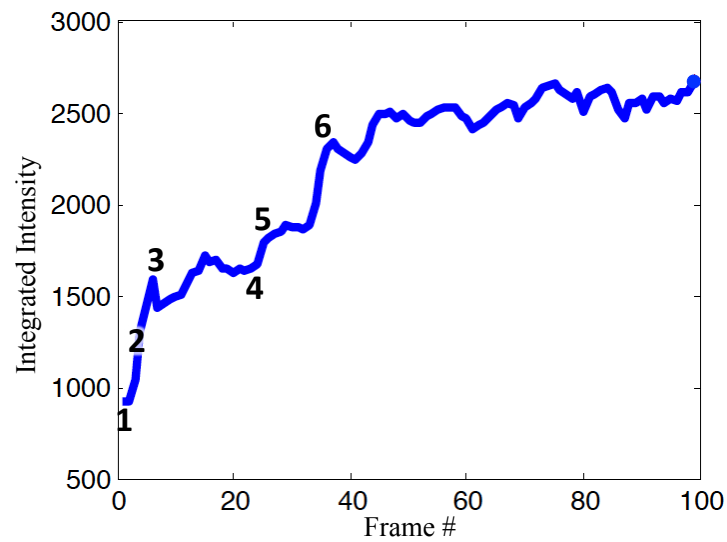

(c) integrated intensity

Figure 9: Motion of a (10.1) peak during relaxation. 

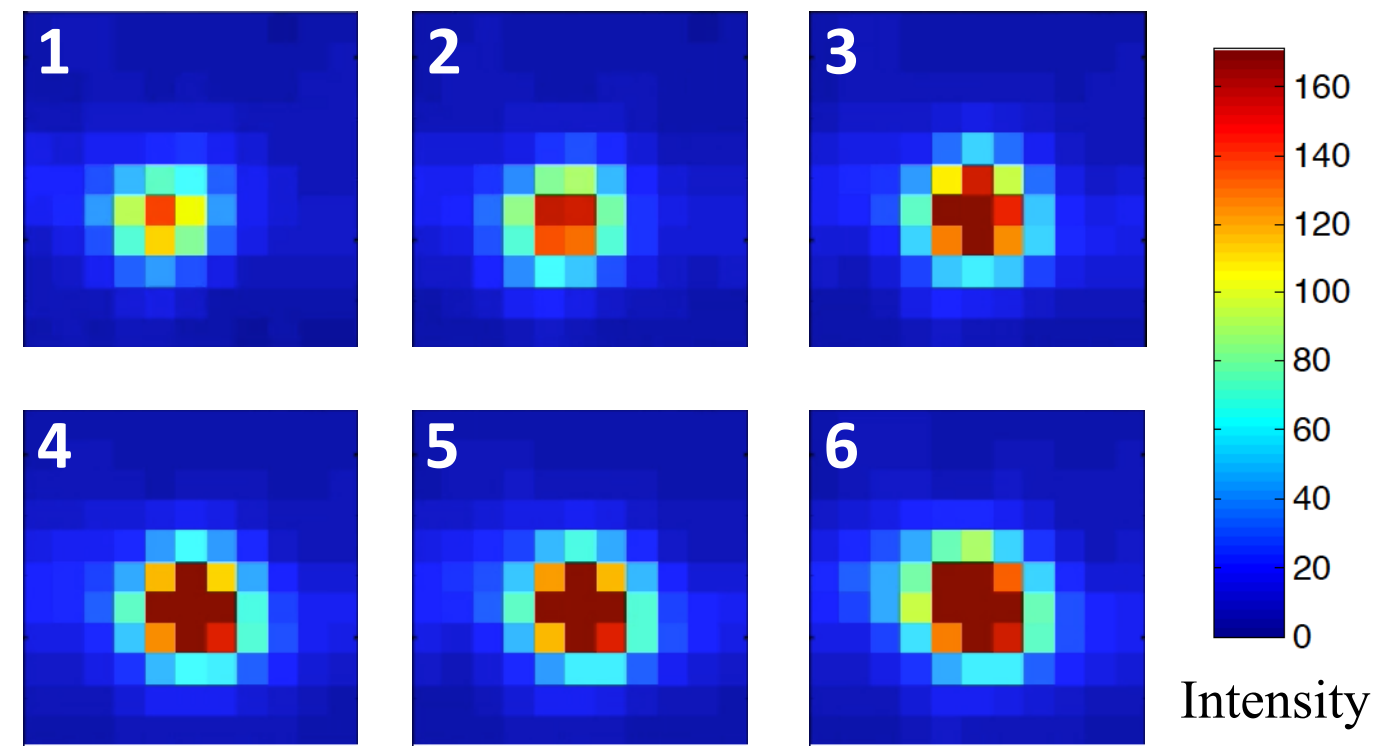

Figure 10: Image of (10.1) peak with numbering corresponding to Figure 9.

In the analysis of strain using a far field detector, accuracy depends on sub-pixel resolution of peak displacement in the radial direction. However, as shown in Figure 9, shifts in radial displacement were frequently accompanied by changes in azimuthal position and integrated intensity. Such abrupt changes in individual peaks may be associated with plastic bursts following from an avalanche of mobile dislocations, leading in turn to orientation changes in the grain and rendering changes in the diffracting volume and concomitant intensity. This would be a manifestation of the collective motion of dislocations within a grain, a possibility indicated by studies of AZ31 and AZ91 as noted in the literature review above [24, 25, 26]. As a specific example, a change such as that observed between 5 and 6 in Figure $10-$ happening relatively late in the relaxation and showing a significant COM position shift on the order of a few tenths of a pixel, along with a $\sim 25 \%$ change in intensity - is indicative of some isolated plastic activity (in this grain or somewhere in the sample). However, there are alternative explanations for the experimental observations that must be considered: through the course of deformation, rigid body motion of the sample could bring grains into (or out of) the diffraction condition, or, discretization errors could be the source of the step-like behavior. Detector technology is advancing rapidly, and the increased accuracy of lattice strain measurement along with greater 
temporal detail will provide for more detailed examination of mechanisms for metal plasticity. The results of the present work provide support for future study of intermittency associated with such mechanisms at the scale of individual grains through high-energy x-ray diffraction.

The technique of high-energy x-ray diffraction provides the capability to isolate aspects of mechanical response at the mesoscale through both the recovery of tensorial lattice strain history on a grain-by-grain basis and temporal response of individual components of strain. The latter offers a means to discern kinetics between slip modes within a grain, which will be key to advancing models for constitutive response at the mesoscale.

\section{Acknowledgments}

This work benefited from support by the US Department of Energy under Contract No. DEFG36-05GO15049. Use of the Advanced Photon Source was supported by the US Department of Energy, Office of Science, Office of Basic Energy Sciences, under Contract No. DE-AC02-06CH11357. We are indebted to Dr. Jonathan Wright of the European Synchrotron Facility for guidance on the use of ImageD11 and Dr. Jonathan Almer of the Advanced Photon Source for assistance in calibration of position and distance for the detector array.

\section{Appendix A. Evaluation of lattice strains}

The measured reciprocal vector associated with reflection $i, \mathbf{g}^{(i)}$, is related to the reciprocal vector in the (undeformed) reference, $\tilde{\mathbf{g}}_{0}^{(i)}$, through the tensor $\tilde{\mathbf{F}}^{-T}$ as $[4,5]$,

$$
\mathbf{g}^{(i)}=\tilde{\mathbf{F}}^{-T} \tilde{\mathbf{g}}_{0}^{(i)}
$$

Using the decomposition $\tilde{\mathbf{F}}=\mathbf{V R}$ and considering the elastic strain to be small,

$$
\mathbf{V}=(\mathbf{I}+\boldsymbol{\epsilon})
$$

where $\mathbf{I}$ is the identity and $\boldsymbol{\epsilon}<<\mathbf{I}$ is the elastic strain, gives

$$
(\mathbf{I}+\boldsymbol{\epsilon}) \mathbf{g}^{(i)}=\mathbf{R} \tilde{\mathbf{g}}_{0}^{(i)}
$$

Defining $\mathbf{g}_{0}=\mathbf{R} \tilde{\mathbf{g}}_{0}$ and rearranging leads to

$$
\epsilon \mathbf{g}^{(i)}=\mathbf{g}_{0}^{(i)}-\mathbf{g}^{(i)}
$$


Taking the inner product with $\mathbf{g}^{(i)}$, then dividing by $\left|\mathbf{g}^{(i)}\right|^{2}$ gives

$$
\hat{\mathbf{g}}^{(i)} \cdot \boldsymbol{\epsilon} \hat{\mathbf{g}}^{(i)}=\hat{\mathbf{g}}^{(i)} \cdot \frac{\mathbf{g}_{0}^{(i)}-\mathbf{g}^{(i)}}{\left|\mathbf{g}^{(i)}\right|}
$$

where the unit vector $\hat{\mathrm{g}}=\frac{\mathrm{g}}{|\mathrm{g}|}$ is used. Equation A.5 leads to an overdetermined system of equations for lattice strain. The iterative solution procedure described in [33] is followed to develop lattice orientation and strain for individual grains.

\section{References}

[1] H. F. Poulsen, Three-Dimensional X-Ray Microscopy, Springer-Verlag, Berlin Heidelberg, 2004.

[2] U. Lienert, M. C. Brandes, J. V. Bernier, J. Weiss, S. D. Shastri, M. J. Mills, M. P. Miller, In situ single-grain peak profile measurements on Ti-7Al during tensile deformation, Materials Science and Engineering A 524 (1-2) (2009) 46-54.

[3] J. Oddershede, S. Schmidt, H. Poulsen, H. Srensen, J. Wright, W. Reimers, Determining grain resolved stresses in polycrystalline materials using three-dimensional x-ray diffraction, Journal of Applied Crystallography 43 (2010) 539549.

[4] J. Edmiston, N. Barton, J. Bernier, G. Johnson, D. Steigmann, Precision of lattice strain and orientation measurements using high-energy monochromatic x-ray diffraction, J. Appl. Crystallogr. 44 (2011) 299.

[5] J. Edmiston, J. Bernier, N. Barton, G. Johnson, Lattice refinement strategies, Acta Crystallogr. A, Found. Crystallogr. A68 (2012) 181.

[6] M. Miller, P. Dawson, Understanding local deformation in metallic polycrystals using high energy x-rays and finite elements, Current Opinion in Solid State and Materials Science (0) (2014) -. doi:http://dx.doi.org/10.1016/j.cossms.2014.09.001.

URL http://www.sciencedirect.com/science/article/pii/S1359028614000576

[7] S. Agnew, O. Duygulu, Plastic anisotropy and the role of non-basal slip in magnesium alloy az31b, International Journal of Plasticity 21 (2005) 1161-1193. 
[8] A. Akhtar, E. Teghtsoonian, Substitutional solution hardening of magnesium single crystals, Philosophical Magazine 25 (4) (1972/04/) 897 916.

[9] A. Akhtar, E. Teghtsoonian, Solid solution strengthening of magnesium single crystals. i. alloying behaviour in basal slip, Acta Metallurgica 17 (11) (1969/11/) $1339-49$.

[10] B. Bhattacharya, M. Niewczas, Work-hardening behaviour of mg single crystals oriented for basal slip, Philosophical Magazine 91 (2011) 22272247.

[11] A. Akhtar, E. Teghtsoonian, Solid solution strengthening of magnesium single crystals. ii. the effect of solute on the ease of prismatic slip, Acta Metallurgica 17 (11) (1969/11/) $1351-6$.

[12] T. Obara, H. Yoshinga, S. Morozumi, $\{11 \overline{2} 2\}\langle\overline{1} \overline{1} 23\rangle$ slip system in magnesium, Acta Metallurgica 21 (1973) 845-853.

[13] X. Zhong, G. Huang, F. He, Q. Liu, Mechanical properties and microstructure of az31 magnesium alloy under uni-axial tensile loading, Materials Science Forum 686 (2011) 219-224.

[14] E. Karimi, A. Zarei-Hanzaki, M. Pishbin, H. Abedi, P. Changizian, Instantaneous strain rate sensitivity of wrought az31 magnesium alloy, Materials and Design 49 (2013) 173-180.

[15] Y. V. R. K. Prasad, K. P. Rao, Hot workability, microstructural control and rate-controlling mechanisms in cast-homogenized az31 magnesium alloy, Advanced Engineering Materials.

[16] P. Lukáč, Z. Trojanova, "stress relaxation in an az31 magnesium alloy", Key Engineering Materials 465 (2011) 101-104.

[17] J. Koike, T. Kobayashi, T. Mukai, H. Watanabe, M. Suzuki, K. Maruyama, K. Higashi, The activity of non-basal slip systems and dynamic recovery at room temperature in fine-grained az31b magnesium alloys, Acta Materialia 51 (2003) 20552065.

[18] T. Kobayashi, J. Koike, T. Mukai, M. Suzuki, H. Watanabe, K. Maruyama, K. Higashi, Anomalous activity of nonbasal dislocations 
in az31 mg alloys at room temperature, Materials Science Forum 419-422 (2003) 231-236.

[19] W. K. Miller, Creep of die cast az91 magnesium at room temperature and low stress, Metallurgical Transactions A 22 (4) (1991) 873-877. doi:10.1007/BF02658997.

URL http://dx.doi.org/10.1007/BF02658997

[20] O. Muránsky, D. Carr, M. Barnett, E. Oliver, P. Šittner, Investigation of deformation mechanisms involved in the plasticity of az31 mg alloy: In situ neutron diffraction and epsc modelling, Materials Science and Engineering A 496 (2008) 14-24.

[21] P. Cizek, B. Hutchinson, A. Ghaderi, M. Barnett, I. Sabirov, Deformation modes and anisotropy in magnesium alloy az31, International Journal of Materials Research 100 (4) (2009) 556 - 63.

URL http://dx.doi.org/10.3139/146.110070

[22] M. R. Barnett, A. Ghaderi, I. Sabirov, B. Hutchinson, Role of grain boundary sliding in the anisotropy of magnesium alloys, Scripta Materialia 61 (3) (2009) $277-280$.

URL http://dx.doi.org/10.1016/j.scriptamat.2009.04.001

[23] H. Wang, P. Wu, C. Tomé, J. Wang, Study of lattice strains in magnesium alloy az31 based on a large strain elastic-viscoplastic self-consistent polycrystal model, International Journal of Solids and Structures 49.

[24] M. Janeček, F. Chmelík, Mechanisms of plastic deformation in az31 magnesium alloy investigated by acoustic emission and electron microscopy, in: F. Czerwinski (Ed.), Magnesium Alloys - Design, Processing and Properties, InTech, 2011.

URL http://www. intechopen.com/books/magnesium-alloys-design-processing-and-pr

[25] C. Corby, C. Cacéras, P. Lukáč, Serrated flow in magnesium alloy az91, Materials Science and Engineering A 387-389 (2004) 22-24.

[26] Z. Trojanova, C. Cacéras, On the strain to the onset of serrated flow in a magnesium alloy, Scripta Materialia 56 (2007) 793-796. 
[27] C. Aydner, J. Bernier, B. Clausen, U. Lienert, C. Tomé, D. Brown, Evolution of stress in individual grains and twins in a magnesium alloy aggregate, PHYSICAL REVIEW B 80 (2009) 024113.

[28] J. Oddershede, B. Camin, S. Schmidt, L. Mikkelsen, H. Sorensen, U. Lienert, H. Poulsen, W. Reimers, Measuring the stress field around an evolving crack in tensile deformed mg az31 using three-dimensional x-ray diffraction, Acta Materialia 60 (8) (2012/05/) 3570 - 80.

[29] A. Hammersley, Esrf internal report, esrf98ha01t, fit2d v9.129 reference manual v3.1 (1998).

[30] http://sourceforge.net/apps/trac/fable/wiki (2012).

[31] S. Schmidt, Grainspotter: a fast and robust polycrystalline indexing algorithm, Journal of Applied Crystallography 47 (2014) 276284.

[32] C. Boehlert, Z. Chen, I. Gutirrez-Urrutia, J. Llorca, M. Prez-Prado, In situ analysis of the tensile and tensile-creep deformation mechanisms in rolled \{AZ31\}, Acta Materialia 60 (4) (2012) 1889 - 1904. doi:http://dx.doi.org/10.1016/j.actamat.2011.10.025.

URL http://www.sciencedirect.com/science/article/pii/S1359645411007300

[33] A. Beaudoin, M. Obstalecki, W. Tayon, M. Hernquist, R. Mudrock, P. Kenesei, U. Lienert, In situ assessment of lattice strain in an al-li alloy, Acta Materialia 61 (2013) 3456-3464.

[34] D. Tromans, Elastic anisotropy of hcp metal crystals and polycrystals, International Journal of Research and Reviews in Applied Sciences.

[35] W. Tang, Stress relaxation of az31 alloy using high energy diffraction microscopy, Master's thesis, University of Illinois at Urbana-Champaign (2014).

[36] http://http://www.mathworks.com/discovery/imageenhancement.html (2014).

[37] J. Li, Dislocation dynamics in deformation and recovery, Canadian Journal of Physics 45 (1967) $403-509$. 
[38] S. Agnew, D. Brown, C. Tomé, Validating a polycrystal model for the elastoplastic response of magnesium alloy az31 using in situ neutron diffraction, Acta Mater. 54 (2006) 48414852. 

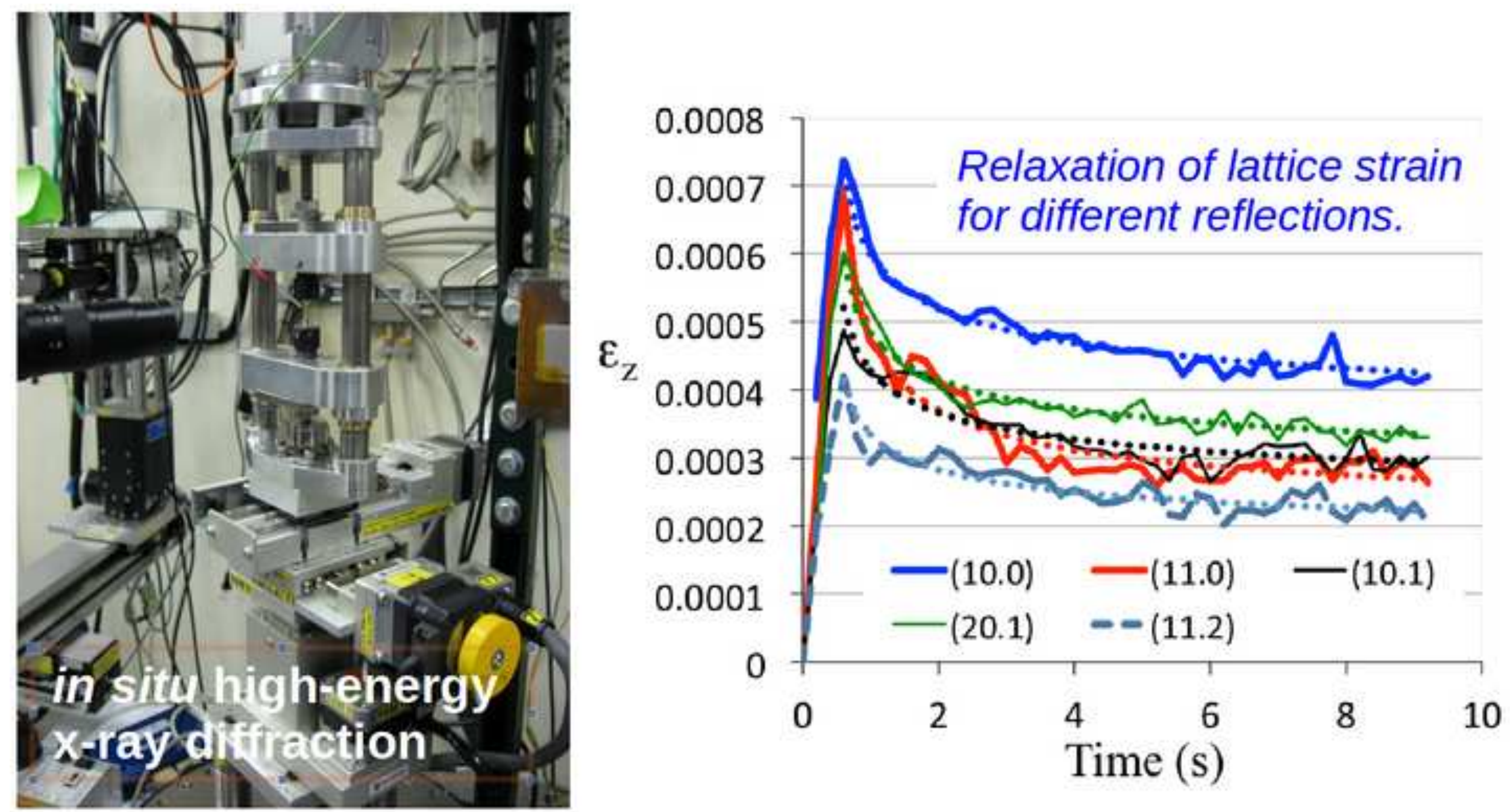